Artículo científico

Volumen 32(3):779-789. Septiembre-diciembre, 2021 e-ISSN 2215-3608, doi:10.15517/am.v32i3.45506 https://revistas.ucr.ac.cr/index.php/agromeso/index

\title{
Colonización endofítica del hongo nematófago Pochonia chlamydosporia en cultivos de Solanaceae y Cucurbitaceae $^{1}$
}

\section{Endophytic colonization of the nematophagous fungus Pochonia chlamydosporia in Solanaceae and Cucurbitaceae crops}

\author{
Wilson Geobel Ceiro-Catasú2, Jersys Arévalo-Ortega ${ }^{3}$,Leopoldo Hidalgo-Díaz ${ }^{3}$
}

1 Recepción: 27 de enero, 2021. Aceptación: 29 de abril, 2021. Este trabajo formó parte de los resultados de una tesis de Doctorado en Ciencias Agrícolas del primer autor, financiado por el Centro Nacional de Sanidad Agropecuaria (CENSA), Cuba.

2 Universidad de Granma, Facultad de Ciencias Agropecuarias, Km 171⁄2 carretera Bayamo-Manzanillo, Bayamo, Granma, Cuba.wceiroc@ gmail.com (autor para la correspondencia, https://orcid.org/0000-0003-2065-2279).

3 Centro Nacional de Sanidad Agropecuaria (CENSA), Dirección de Sanidad Vegetal, Apartado 10, San José de las Lajas, Mayabeque, Cuba. jersys@censa.edu.cu (https://orcid.org/0000-0002-5630-7314), leopoldoj.hidalgo@gmail.com (https://orcid.org/0000-0001-9083-1825).

\section{Resumen}

Introducción. Las especies fúngicas del género Pochonia son importantes para el manejo de nematodos fitoparásitos. Algunas de ellas colonizan en forma endófita a sus hospedantes vegetales y les proporcionan adaptabilidad, bio-estimulación vegetal e inducción de resistencia; dichas ventajas son esenciales para la selección de cepas a utilizar en el bio-manejo de fitonematodos. Objetivo. Evaluar la colonización endofítica y la bio-estimulación vegetal por las cepas nematófagas IMI SD 187 y 193 de P. chlamydosporia sobre especies hortícolas de las familias Solanaeceae y Cucurbitaceae. Materiales y métodos. El experimento se realizó dentro de invernaderos en el Centro Nacional de Sanidad Agropecuaria, provincia Mayabeque, Cuba. Se utilizaron dos cepas de P. chlamydosporia, denominadas IMI SD 187 y 193, así como tomate (S. lycopersicum) cv. HA 3057, pimiento (Capsicum annuum L.) cv. Grandísimo, pepino (Cucumis sativus L.) cv. INIVIT P 2007 y melón (Cucumis melo L.) cv. Zest F1. En ambas cepas se evaluó la colonización endofítica, del sustrato y de la raíz, así como algunos parámetros del crecimiento de las plantas. Resultados. Las cepas evaluadas colonizaron entre 3 y $16 \%$ el interior de las raíces. La cepa IMI SD 187 colonizó a todas las especies evaluadas, mientras que 193 no pudo colonizar al melón; sin embargo, ambas cepas colonizaron la raíz y el sustrato con valores entre $2 \times 10^{3}$ y $2 \times 10^{4} \mathrm{UFC} \mathrm{g}^{-1}$, respectivamente. IMI SD 187 alcanzó los mayores valores de bio-estimulación en longitud y masa fresca de las raíces en pimiento. Conclusión. Las dos cepas autóctonas de $P$. chamydosporia colonizaron a las especies estudiadas y la mayor bio-estimulación del crecimiento se obtuvo con IMI SD 187 en el cultivar de pimiento.

Palabras clave: bio-estimulación vegetal, cepas autóctonas, hongos endófitos, hortalizas.
Abstract
Introduction. The fungal species of the genus Pochonia are important for the management of phytoparasitic nematodes. Some of them endophyte colonize their plant hosts and provide adaptability, plant bio-stimulation, and 
resistance induction; these advantages are essential for the selection of strains to be used in the bio-management of phytonematodes. Objective. To evaluate the endophytic colonization and plant bio-stimulation by the nematophagous strains IMI SD 187 and 193 of $P$. chlamydosporia on horticultural species of the Solanaeceae and Cucurbitaceae families. Materials and methods. The experiment was carried out inside greenhouses at the National Center for Animal and Plant Health, Mayabeque province, Cuba. Two strains of $P$. chlamydosporia were used, named IMI SD 187 and 193, as well as cultivars of tomato (S. lycopersicum) cv. HA 3057, pepper (Capsicum annuum L.) cv. Grandisimo, cucumber (Cucumis sativus L.) cv. INIVIT P 2007, and melon (Cucumis melo L.) cv. Zest F1. In both strains, endophytic, substrate, and root colonization, as well as some plant growth parameters were evaluated. Results. The evaluated strains colonized between 3 and $16 \%$ of the interior of the roots. The IMI SD 187 strain colonized all the evaluated species, while 193 could not colonize the melon; however, both strains colonized the root and the substrate with values between $2 \times 10^{3}$ and $2 \times 10^{4} \mathrm{CFU} \mathrm{g}^{-1}$, respectively. IMI SD 187 reached the highest bio-stimulation values in length and fresh mass of the roots in pepper. Conclusion. The two native strains of $P$. chlamydosporia colonized the studied species and the highest bio-stimulation of growth was obtained with IMI SD 187 in the pepper cultivar.

Keywords: plant bio-stimulation, autochthonous strains, endophyte fungi, vegetables.

\section{Introducción}

Los hongos nematófagos son de gran importancia para el manejo de nematodos fitoparásitos en cultivos de interés económico; entre estos se incluyen los que se caracterizan por ser endófitos, los cuales confieren ventajas a sus hospedantes vegetales como una mejor adaptabilidad ecológica, potenciación de la síntesis de fitohormonas, bioestimulación en la promoción del crecimiento vegetal, inducción de resistencia sistémica, síntesis de metabolitos secundarios frente a plagas y tolerancia al estrés abiótico (Latz et al., 2018; Nassimi \& Taheri, 2017).

Bajo el término endófito se agruparon aquellos microorganismos que habitaban dentro de las plantas, pero luego se incluyó a todos aquellos que viven dentro de los tejidos vegetales, a excepción de los hongos formadores de micorrizas. Una característica común de estos es la formación de relaciones bióticas muy complejas e intensas con sus hospedantes vegetales (Shah et al., 2019).

Pochonia chlamydosporia además de colonizar el suelo y el sustrato, es un endófito natural de raíces de plantas monocotiledóneas (Maciá-Vicente et al., 2009). Este hongo se conoce por sus efectos en el biocontrol de patógenos de las raíces y de nematodos fitoparásitos; es capaz de colonizar las raíces de las plantas a nivel endofítico y sobrevivir dentro de estas ante situaciones de estrés biótico o abiótico presentes en su hábitat natural (HidalgoDíaz \& Ceiro, 2017; Hidalgo-Díaz et al., 2017). Su supervivencia en el interior de las raíces es una interacción beneficiosa y tiene efectos en el manejo de nematodos formadores de agallas del género Meloidogyne, debido a que este fitoparásito produce sus masas de huevos sobre las raíces infestadas, lo que posibilita la actividad colonizadora y parasítica de P. chlamydosporia (Topalovi'c et al., 2020).

Se ha demostrado la colonización endofítica de P. chlamydosporia sobre Hordeum vulgare L. y Solanum lycopersicum L. (Bordallo et al., 2002; López-Llorca et al., 2002; López-Llorca \& Maciá-Vicente 2009). Además, se evidenció la capacidad de colonización endofítica de la cepa IMI SD 187 en cultivares de plátanos, bananos y frijoles en Cuba, así como, su efecto bioestimulador del crecimiento vegetal (Arévalo et al., 2019; Hernández et al., 2016).

Los exudados de las raíces, así como las especies vegetales que los producen, son elementos esenciales para que P. chlamydosporia logre la colonización del suelo o sustrato (Hidalgo-Díaz et al., 2017). Este hongo nematófago en interacción con una fertilización nitrogenada, estimuló el crecimiento en altura de Phaseolus vulgaris L., debido al 
incremento de los contenidos de clorofila, proteínas totales, nitrógeno, fósforo y actividad enzimática de la nitrato reductasa de las plantas; a su vez, el hongo fue capaz de reducir el número de agallas y masas de huevos de $M$. incognita (Rushda \& Ambreen, 2014).

Un incremento de $36 \%$ del crecimiento vegetal en plantas de S. lycopersicum infestadas con Meloidogyne incognita por efecto de P. chlamydosporia, fue obtenido por Siddiqui \& Akhtar (2008). Un estudio más reciente demostró $\geq 20 \%$ de estimulación del crecimiento vegetal en plantas de tomate tratadas con P. chlamydosporia var. chlamydosporia (Rushda \& Ambreen, 2014).

La presente investigación tuvo como objetivo evaluar el potencial de colonización endofítica y de bioestimulación vegetal de las cepas autóctonas nematófagas IMI SD 187 y 193 de P. chlamydosporia sobre especies hortícolas de las familias Solanaeceae y Cucurbitaceae.

\section{Materiales y métodos}

\section{Localización y establecimiento del experimento}

La investigación se realizó en el periodo de enero-marzo de 2017 en un invernadero en el Centro Nacional de Sanidad Agropecuaria (CENSA), provincia Mayabeque, Cuba. Se utilizaron semillas certificadas de dos solanáceas, tomate (S. lycopersicum) cv. HA 3057, pimiento (Capsicum annuum L.) cv. Grandísimo, así como dos cucurbitáceas, pepino (Cucumis sativus L.) cv. INIVIT P 2007 y melón (Cucumis melo L.) cv. Zest F1. Las semillas se desinfectaron con $\mathrm{NaOCl}$ al $1 \%$ durante cinco minutos, se sembraron dos por cada maceta que contenía $300 \mathrm{~g}$ del sustrato compuesto por suelo Fluvisol Típico y humus de lombriz (3:1v/v) esterilizado.

Al momento de la siembra se inocularon al sustrato $5 \times 10^{3}$ clamidosporas $\mathrm{g}^{-1}$ de ambas cepas de $P$. chalmydosporia . Las cepas autóctonas evaluadas fueron IMI SD 187 ( $P$. chlamydosporia var. catenulata, bioproducto KlamiC ${ }^{\circledR}$, CENSA, Cuba) y 193 (P. chlamydosporia var. chlamydosporia), ambas obtenidas del cepario de conservación del laboratorio de micología del CENSA y multiplicadas en condiciones controladas mediante fermentación en estado sólido en bolsas (Montes de Oca et al., 2009). El inóculo que se aplicó tenía una concentración de clamidosporas de $\geq 1,20 \times 10^{7} \mathrm{~g}^{-1}$ y una viabilidad de $\geq 94 \%$.

Posterior a la germinación de las semillas se dejó una sola planta por cada maceta, mantenidas dentro del invernadero a temperatura ambiente durante treinta días; en el caso de la especie de C. аппиит demoró su germinación por quince días más, debido al lento crecimiento del cultivar utilizado. Las evaluaciones se realizaron al final de ambos períodos, como se expondrá a continuación.

\section{Indicadores evaluados}

La colonización del sustrato se determinó de acuerdo con el método propuesto por Kerry \& Bourne (2002). Las placas resultantes se incubaron en la oscuridad a una temperatura de $25{ }^{\circ} \mathrm{C}$, luego de transcurridos veintiún días se calculó la cantidad de unidades formadoras de colonias por gramo ( $\left.\mathrm{UFC} \mathrm{g}^{-1}\right)$ de sustrato.

Para determinar la colonización endofítica se utilizó el método descrito por Maciá-Vicente et al. (2009), con el fin de constatar que el crecimiento de P. chlamydosporia proviniera del interior y no de la superficie de las raíces. Cada raíz se desinfectó con $\mathrm{NaOCl}(1 \%)$ durante un minuto, luego se enjuagaron tres veces con abundante agua destilada esterilizada, se eliminó el exceso de humedad con papel de filtro esterilizado y se seccionaron en tres porciones (inferior, media y superior). De ellas, se sembraron cuatro segmentos con longitud de $1 \mathrm{~cm}$ en placas que contenían medio semiselectivo (MSS) (Kerry \& Bourne, 2002). Previo a la siembra de los mismos se realizó imprint; para ello, se usaron placas que contenían MSS, sobre estas se depositaron los segmentos a sembrar durante 
$5 \mathrm{~s}$. Las placas se sellaron con parafilm y se incubaron a temperatura constante de $25^{\circ} \mathrm{C}$ en la oscuridad durante 14 días, al cabo de ese tiempo se cuantificaron los segmentos con presencia de crecimiento fúngico endófito y el resultado se expresó en porcentaje.

Para evidenciar la colonización endofítica, se procedió a clarificar raíces secundarias con $\mathrm{KOH}$ al $10 \%$ durante 10 min, luego se tiñeron con azul de algodón, se enjuagaron con abundante agua destilada y se montaron en porta objetos para observarlas al microscopio.

A cada planta se le evaluó la cantidad de hojas activas, mediante conteo directo. La altura de la planta $(\mathrm{cm})$, y la longitud de la raíz $(\mathrm{cm})$, se midieron con una regla milimetrada, la primera desde la base del tallo hasta el meristemo apical; mientras la segunda se midió desde el cuello de la raíz hasta la cofia. La masa fresca foliar (g) y la masa fresca de raíces (g) se obtuvieron en una balanza analítica (SARTORIUS BP 310 S).

\section{Diseño experimental y procesamiento estadístico}

Se utilizó un diseño completamente aleatorizado con arreglo factorial, el Factor I las cuatro especies vegetales y el Factor II las dos cepas autóctonas de P. chlamydosporia. Los tratamientos se obtuvieron mediante la combinación de las cepas con cada especie vegetal y los respectivos controles sin tratar; de esta forma se conformaron ocho tratamientos con cinco réplicas cada uno. Los datos se procesaron mediante ANOVA factorial y las medias se compararon con la prueba de comparación múltiple de Tukey $(\mathrm{p} \leq 0,05)$, para ello, se utilizó el paquete InfoSat (Di Rienzo et al., 2008). Previo a la realización del análisis estadístico, los valores expresados en porcentaje se transformaron mediante ARCSEN $\sqrt{x} / 100$; mientras que se usó $\mathrm{LOG}_{10} \mathrm{x}+1$ para las UFC de P. chlamydosporia.

\section{Resultados}

\section{Niveles de colonización endofítica por efecto de las cepas autóctonas de $P$. chlamydosporia en especies de las familias Solanaceae y Cucurbitaceae}

Al analizar la colonización endofítica se evidenció una mayor colonización en las interacciones C. sativus-cepa 193, C. sativus-cepa IMI SD 187, S. lycopersicum-cepa IMI SD 187 y C. melo-cepa IMI SD 187, las cuales no se diferenciaron significativamente entre sí, pero a su vez, difirieron con los tratamientos que expresaron menores valores de colonización como $S$. lycopersicum-cepa 193, C. annuиm-cepa 193 у C. апnиит-cepa IMI SD 187. La interacción C. melo-cepa 193 no mostró actividad endofítica colonizadora (Figuras 1 y 2). Se demostró que la cepa IMI SD 187 colonizó el interior de las raíces de todas las especies vegetales estudiadas, sin embargo, la cepa 193 no logró colonizar a C. melo.

\section{Actividad saprofítica de las cepas autóctonas de P. chlamydosporia en especies de las familias Solanaceae y Cucurbitaceae}

La colonización de las raíces tuvo diferencias significativas (Tukey, $\mathrm{p} \leq 0,05$ ) entre los tratamientos. Los mayores valores, sin diferencias significativas entre sí, se registraron en las interacciones C. sativus-IMI SD 187, C. sativus-193, S. lycopersicum-IMI SD 187, S. lycopersicum-193, C. melo-IMI SD 187, C. melo-193 у C. аппиит-IMI SD 187. La combinación C. аппиит-193 registró la menor colonización diferenciándose del resto de las interacciones (Figura 3). 


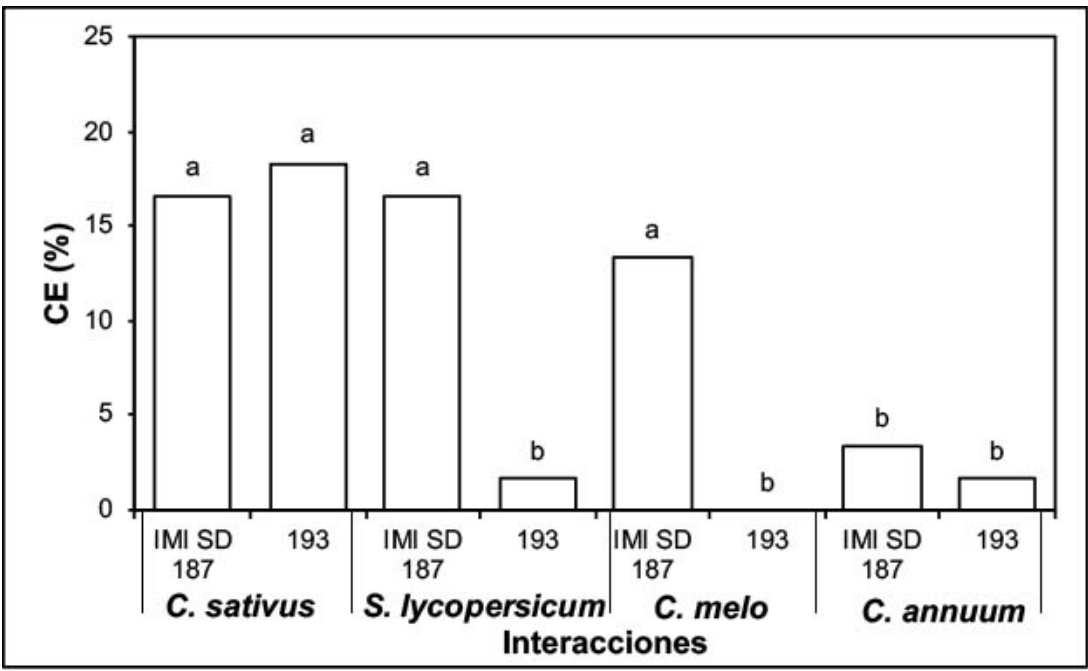

Figura 1. Colonización endofítica por efecto de dos cepas de P. chlamydosporia sobre S. lycopersicum, C. annuum, C. sativus y C. melo. Laboratorio de Micología, Centro Nacional de Sanidad Agropecuaria (CENSA), Mayabeque, Cuba. 2017.

Letras distintas evidencian diferencias significativas (Tukey, $\mathrm{p} \leq 0,05$ ). CE: colonización endofítica. Error estándar: 0,68.

Figure 1. Endophytic colonization due to the effect of two strains of P. chlamydosporia on S. lycopersicum, C. annuum, C. sativus, and C. melo. Mycology Laboratory, National Center for Animal and Plant Health (CENSA), Mayabeque, Cuba. 2017.

Different letters on bars show significant differences (Tukey, $\mathrm{p} \leq 0.05$ ). CE: endophytic colonization. Standard error: 0.68.
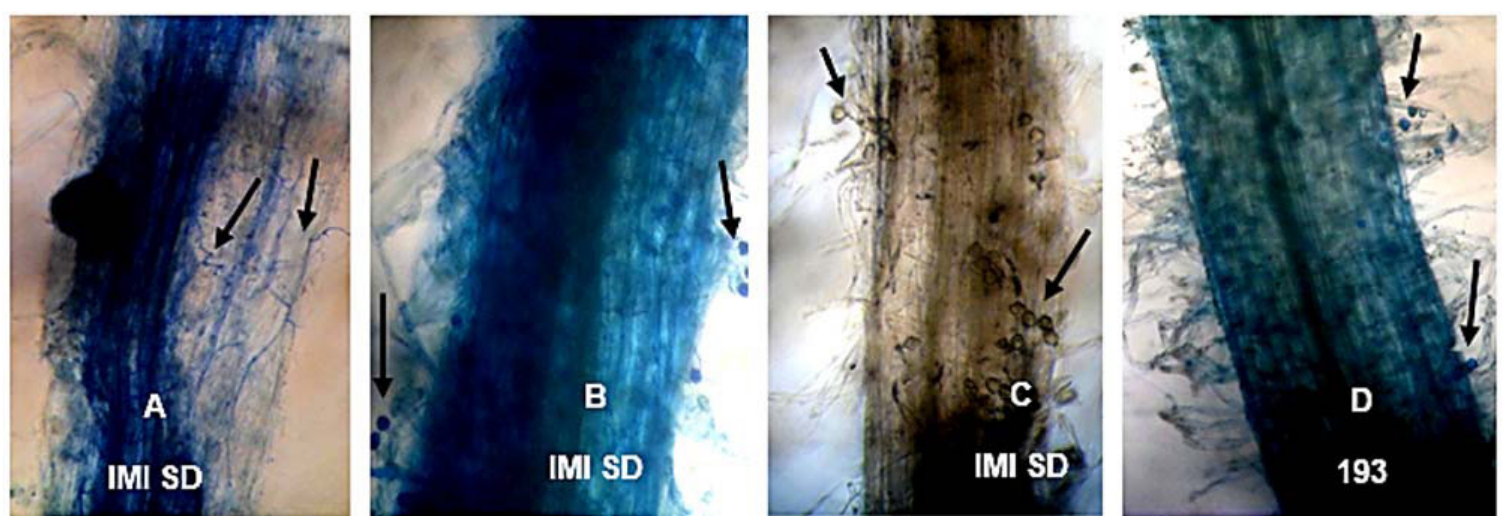

Figura 2. Colonización endofítica y actividad rizosférica de $P$. chlamydosporia. Las flechas muestran hifas con desarrollo endófito en raíces de $C$. sativus (A), la presencia de clamidosporas en el sistema radical de $S$. lycopersicum (B), C. melo (C) y C. annuum (D). Laboratorio de Micología, Centro Nacional de Sanidad Agropecuaria (CENSA), Mayabeque, Cuba. 2017.

Figure 2. Endophytic colonization and rhizospheric activity of P. chlamydosporia. The arrows show hyphae with endophytic development in C. sativus roots (A), presence of chlamydospores in the root system of S. lycopersicum (B), C. melo (C) and C. annuum (D). Mycology Laboratory, National Center for Animal and Plant Health (CENSA), Mayabeque, Cuba. 2017. 


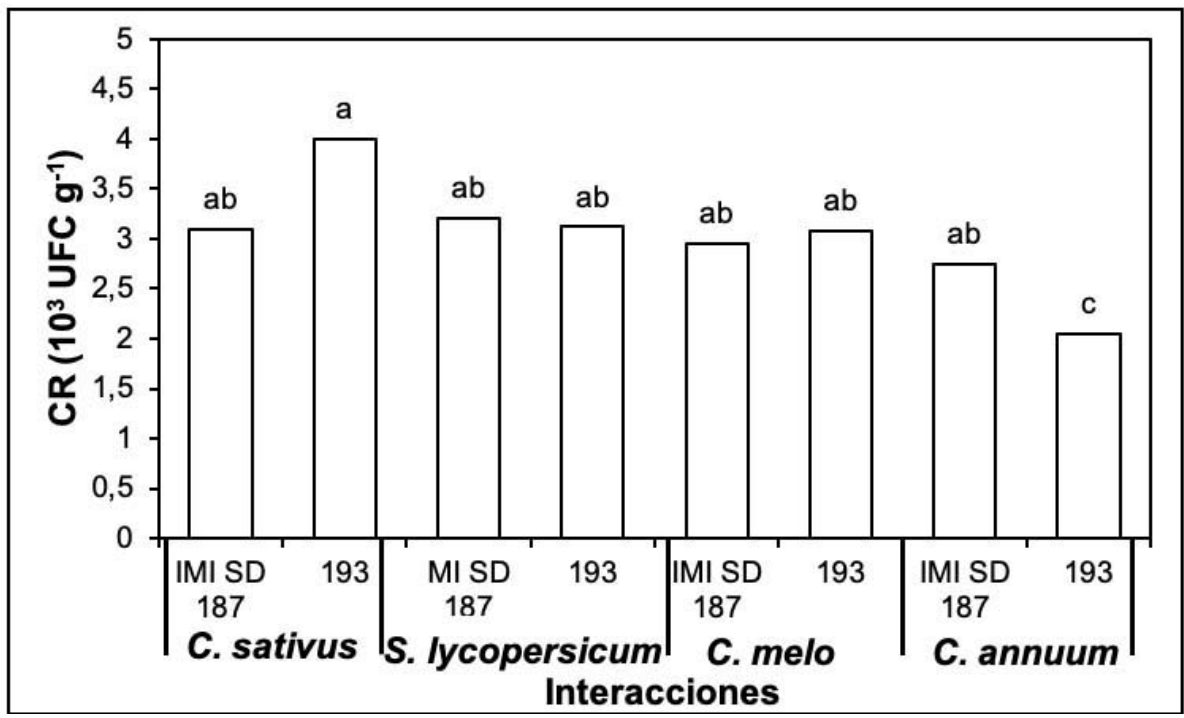

Figura 3. Colonización de raíces por efecto de las dos cepas de P. chlamydosporia en S. lycopersicum, C. annuum, C. sativus y C.melo. Laboratorio de Micología, Centro Nacional de Sanidad Agropecuaria (CENSA), Mayabeque, Cuba. 2017.

Letras distintas sobre las barras evidencian diferencias significativas (Tukey, p $\leq 0,05$ ). CR: colonización de la raíz. Error estándar: 0,09.

Figure 3. Root colonization by the effect of the two strains of P. chlamydosporia on S. lycopersicum, C. annuum, C. sativus and C. melo. Mycology Laboratory, National Center for Animal and Plant Health (CENSA), Mayabeque, Cuba. 2017.

Different letters on the bars show significant differences (Tukey, $\mathrm{p} \leq 0.05$ ). CR: root colonization. Standard error: 0.09.

Respecto a la colonización del sustrato, los mayores valores correspondieron a los tratamientos donde se inocularon ambas cepas en $C$. sativus y $S$. lycopersicum. Los menores valores se registraron en $C$. annuит у $C$. melo inoculados con las dos cepas evaluadas, sin diferencias con las interacciones S. lycopersicum-cepas IMI SD 187 y 193, así como C. sativus-cepa IMI SD 187 (Figura 4).

\section{Bio-estimulación del crecimiento vegetal por efecto de las cepas autóctonas de $\boldsymbol{P}$. chlamydosporia en especies de las familias Solanaceae y Cucurbitaceae}

El crecimiento vegetal mostró diferencias significativas entre tratamientos en las variables: cantidad de hojas, longitud de las raíces y masa fresca radical (Tukey, p $\leq 0,05$ ). La cepa IMI SD 187 presentó diferencias comparada con las plantas no inoculadas en longitud de raíces en $C$. melo, mientras que no se constataron diferencias entre cepas nematófagas. En las plantas de C. аппиит inoculadas con las dos cepas autóctonas de $P$. chlamydosporia se contaron las mayores cantidades de hojas, comparado con las plantas no inoculadas en este mismo cultivo. La masa fresca de las raíces mostró diferencias entre IMI SD 187 y las plantas no inoculadas, pero no se diferenciaron entre sí con el control y la cepa 193, así como con IMI SD 187 y 193. Entre los restantes tratamientos no se registraron diferencias significativas (Cuadro 1). 


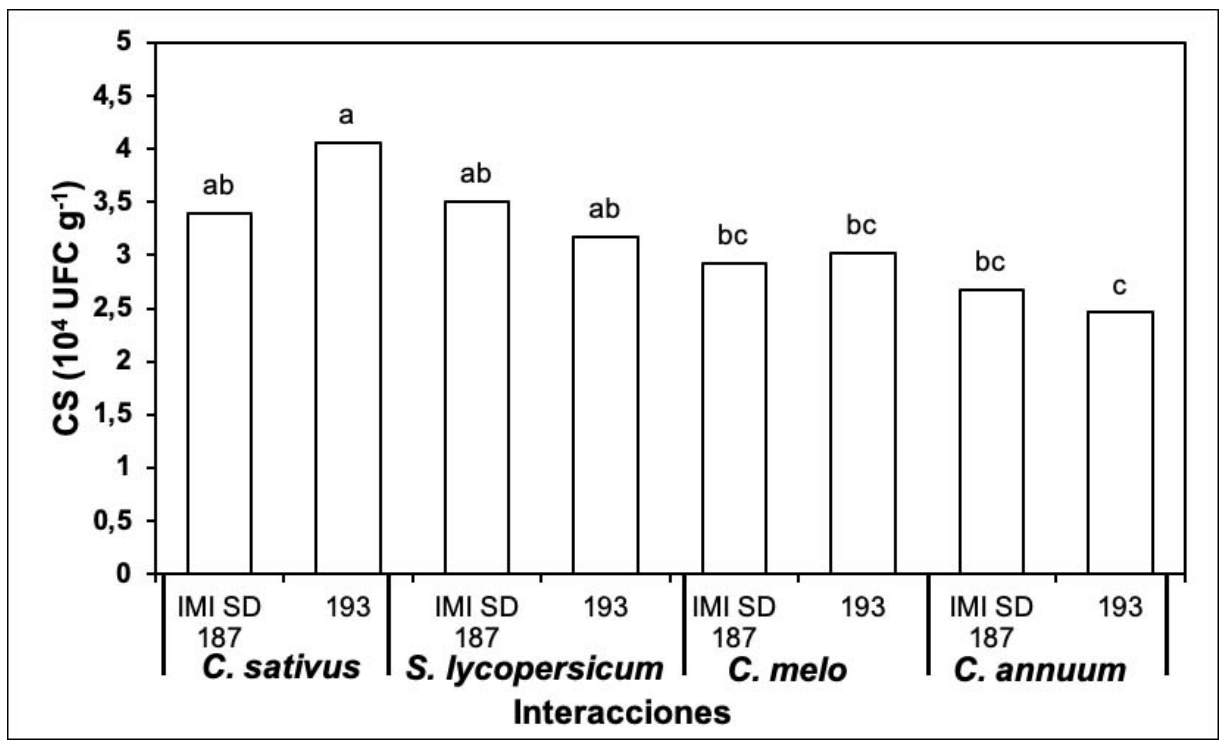

Figura 4. Colonización del sustrato de dos cepas de P. chlamydosporia sobre S. lycopersicum, C. annuum, C. sativus y C. melo. Laboratorio de Micología, Centro Nacional de Sanidad Agropecuaria (CENSA), Mayabeque, Cuba. 2017.

Letras distintas sobre las barras evidencian diferencias significativas (Tukey, p $\leq 0,05$ ). CS: colonización del sustrato. Error estándar: 0,03 .

Figure 4. Colonization of the substrate of two strains of P. chlamydosporia on S. lycopersicum, C. annuum, C. sativus, and C. melo. Mycology Laboratory, National Center for Animal and Plant Health (CENSA), Mayabeque, Cuba. 2017.

Different letters on the bars show significant differences (Tukey, $\mathrm{p} \leq 0.05$ ). CS: colonization of the substrate. Standard error: 0.03 .

Cuadro 1. Efecto de dos cepas de P. chlamydosporia sobre las variables del crecimiento de S. lycopersicum, C. annuum, C. sativus y C. melo. Laboratorio de Micología, Centro Nacional de Sanidad Agropecuaria (CENSA), Mayabeque, Cuba. 2017.

Table 1. Effect of two strains of P. chlamydosporia on growth indicators of S. lycopersicum, C. annuum, C. sativus, and C. melo. Mycology Laboratory, National Center for Animal and Plant Health (CENSA), Mayabeque, Cuba. 2017.

\begin{tabular}{|c|c|c|c|c|c|c|}
\hline Cultivos & Cepas & $\mathbf{C H}$ & $\mathbf{A P}(\mathbf{c m})$ & MFF (g) & LR (cm) & MFR (g) \\
\hline \multirow{3}{*}{$\begin{array}{l}\text { C. sativus cv. } \\
\text { INIVIT P } 2007\end{array}$} & IMI SD 187 & $6,80 \mathrm{a}$ & 38,32 a & 10,19 a & $6,00 \mathrm{a}$ & $0,70 \mathrm{a}$ \\
\hline & 193 & $6,20 \mathrm{a}$ & $37,26 \mathrm{a}$ & $10,75 \mathrm{a}$ & $5,40 \mathrm{a}$ & $0,52 \mathrm{a}$ \\
\hline & Control & $6,60 \mathrm{a}$ & 38,70 a & $12,07 \mathrm{a}$ & $5,86 \mathrm{a}$ & $0,74 \mathrm{a}$ \\
\hline \multirow{3}{*}{$\begin{array}{l}\text { S. lycopersicum } \\
\text { cv. HA } 3057\end{array}$} & IMI SD 187 & $3,60 \mathrm{a}$ & $11,87 \mathrm{a}$ & $2,06 \mathrm{a}$ & $6,10 \mathrm{a}$ & $0,37 \mathrm{a}$ \\
\hline & 193 & $3,40 \mathrm{a}$ & $11,62 \mathrm{a}$ & $1,57 \mathrm{a}$ & $4,74 \mathrm{a}$ & $0,19 \mathrm{a}$ \\
\hline & Control & $3,20 \mathrm{a}$ & $11,30 \mathrm{a}$ & $1,56 \mathrm{a}$ & $4,62 \mathrm{a}$ & $0,20 \mathrm{a}$ \\
\hline \multirow{3}{*}{$\begin{array}{l}\text { C. melo cv. Zest } \\
\text { F1 }\end{array}$} & IMI SD 187 & $4,40 \mathrm{a}$ & $16,36 \mathrm{a}$ & $5,72 \mathrm{a}$ & $6,74 \mathrm{a}$ & $0,25 \mathrm{a}$ \\
\hline & 193 & $4,20 \mathrm{a}$ & $16,12 \mathrm{a}$ & $6,95 \mathrm{a}$ & $5,74 \mathrm{ab}$ & $0,34 \mathrm{a}$ \\
\hline & Control & $4,40 \mathrm{a}$ & $14,88 \mathrm{a}$ & $5,63 \mathrm{a}$ & $4,86 \mathrm{~b}$ & $0,20 \mathrm{a}$ \\
\hline \multirow{3}{*}{$\begin{array}{l}\text { C. annuum cv. } \\
\text { Grandísimo }\end{array}$} & IMI SD 187 & $5,00 \mathrm{a}$ & $7,30 \mathrm{a}$ & $1,47 \mathrm{a}$ & $6,30 \mathrm{a}$ & $0,63 \mathrm{a}$ \\
\hline & 193 & $5,00 \mathrm{a}$ & $6,08 \mathrm{a}$ & $1,15 \mathrm{a}$ & $5,96 \mathrm{a}$ & $0,50 \mathrm{ab}$ \\
\hline & Control & $3,00 \mathrm{~b}$ & $5,36 \mathrm{a}$ & $0,78 \mathrm{a}$ & $5,90 \mathrm{a}$ & $0,28 \mathrm{~b}$ \\
\hline EE & - & 0,24 & 0,74 & 0,75 & 0,42 & 0,63 \\
\hline
\end{tabular}

CH: cantidad de hojas, AP: altura de las plantas, MFF: masa fresca foliar, LR: longitud de la raíz, MFR: masa fresca radical, EE: error estándar. Letras distintas en las columnas evidencian diferencias significativas (Tukey, $\mathrm{p} \leq 0,05)$ / $\mathrm{CH}$ : number of leaves, AP: plant height, MFF: fresh foliar mass, LR: root length, MFR: fresh root mass, EE: standard error. Different letters in the columns show significant differences (Tukey, $\mathrm{p} \leq 0.05$ ). 


\section{Discusión}

El proceso de colonización endofítica y del sustrato por las cepas autóctonas evaluadas, se desarrolló bajo los efectos de altos contenidos de MO $(29,20 \%)$ en el sustrato, lo cual no coincidió con los resultados expuestos por Kerry \& Hirsch (2011), quienes expusieron que un contenido alto de MO en el suelo afectaba la actividad saprofítica de $P$. chlamydosporia var. chlamydosporia, lo cual no fue observado en esta investigación. El resultado pone de manifiesto que la cepa autóctona cubana IMI SD 187 de la variedad catenulata, colonizó el interior de las raíces de todas las especies vegetales evaluadas en presencia de altos contenidos de MO, lo cual puede atribuirse a que esta cepa fue aislada de un agroecosistema orgánico de cafeto en la región montañosa oriental de Cuba, donde existen suelos ricos en fertilidad (Gutiérrez et al., 2018).

La colonización endofítica observada en la presente investigación varió entre 3 y $16 \%$, lo cual demuestra el potencial colonizador de este hongo en cultivos dicotiledóneos de las familias Solanaceae y Cucurbitaceae. Se considera al interior de las raíces como un nicho ecológico que le permite sobrevivir a la especie fúngica cuando existan condiciones de estrés en la rizosfera. No se constataron trabajos científicos publicados que tuvieran relación con endofitismo en especies de la familia Cucurbitaceae, esta investigación podría ser el primer informe científico sobre el tema. Sin embargo, un estudio anterior registró un rango de colonización entre 4 y $20 \%$ por efecto de la cepa IMI SD 187 en especies de la familia Musaceae (Hernández et al., 2016). Hubo niveles de colonización endofítica de $P$. chlamydosporia var. chlamydosporia en una especie vegetal monocotiledónea, aunque su desarrollo endofítico fue limitado hacia los tejidos más profundos del sistema de raíces (Maciá-Vicente et al., 2009).

Con relación a las especies que registraron menor colonización endofítica, pudo ser causado por una activación de los mecanismos de defensas de la planta, debido a la presencia del hongo nematófago; se impidió, de este modo, su entrada al interior de los tejidos radicales. Hay evidencias de que $P$. chlamydosporia redujo su actividad de colonización rizosférica en plantas de papa por efecto del ácido jasmónico, sustancia involucrada en la activación defensiva de las plantas, pero mantuvo estable su actividad parasítica sobre Meloidogyne chitwoodi (Vieira-DosSantos et al., 2014).

El estímulo en la longitud de la raíz en $C$. melo y la masa fresca de las raíces en $C$. annuum por efecto de las cepas de $P$. chlamydosporia, constituye una ventaja fisiológica para las plantas en cuanto a la absorción de agua y nutrientes, aspecto demostrado por Avelar et al. (2018) en el proceso de nutrición de S. lycopersicum. La estimulación del desarrollo vegetal en presencia de las cepas de $P$. chlamydosporia, puede atribuirse a la síntesis de sustancias orgánicas promotoras del crecimiento vegetal. Al respecto, Vyas et al. (2008) demostraron que en las plántulas de Feronia limonia L. colonizadas con la especie fúngica Piriformospora indica Verma, Varma, Kost, Rexer y Frankense incrementaron los contenidos de proteínas totales y otras sustancias con efecto potenciador del crecimiento vegetal. Ambas cepas de P. chlamydosporia lograron colonizar a la totalidad de las especies vegetales evaluadas y la colonización radical osciló entre 2 y $4 \times 10^{3} \mathrm{UFC} \mathrm{g}^{-1}$, dichos valores fueron superiores a los registrados por Hidalgo-Díaz \& Ceiro (2017), quienes obtuvieron rangos entre 1 y $3 \times 10^{3} \mathrm{UFC} \mathrm{g}^{-1}$ de raíz. También se demostró que poblaciones del hongo nematófago $\geq 10^{3} \mathrm{UFC} \mathrm{g}^{-1}$ de raíz, son suficientes para una efectiva actividad parasítica sobre los nematodos formadores de agallas (Ghahremani et al., 2019).

Las dos cepas autóctonas evaluadas lograron colonizar el sustrato en todas las interacciones evaluadas. Este indicador varió entre 2 y $4 \times 10^{4} \mathrm{UFC} \mathrm{g}^{-1}$, dichos valores superaron los informados por Arévalo et al. (2019), que registraron un rango de colonización de la cepa IMI SD 187 de 0,74-2,06x $10^{4} \mathrm{UFC} \mathrm{g}^{-1}$ de suelo. También se obtuvo, con esta misma cepa, de 2 a $3 \times 10^{4} \mathrm{UFC} \mathrm{g}^{-1}$ de suelo en presencia de estrés salino moderado (Hidalgo-Díaz \& Ceiro, 2017). Cantidades iguales o superiores a $10^{3} \mathrm{UFC} \mathrm{g}^{-1} \mathrm{de} P$. chlamydosporia en los suelos de forma natural, favorecen la formación de los denominados suelos supresores de nematodos, los cuales no requieren de medidas adicionales de manejo para esta plaga (Uddin et al., 2019). 
Se observó que las especies vegetales analizadas experimentaron diferentes respuestas de colonización endofítica por efecto de las cepas autóctonas de P. chlamydosporia. Una mayor colonización endofítica, no fue proporcional a un mayor crecimiento vegetal. C. аппиит con menores niveles de colonización al interior de las raíces, alcanzó la mayor bio-estimulación en cantidad de hojas y masa fresca radical; a diferencia de esta especie, con mayores porcentajes de colonización endofítica, C. sativus no logró estimulación significativa del crecimiento bajo las condiciones experimentales evaluadas. Lo expuesto, sugiere la necesidad de realizar futuras investigaciones sobre esta temática, que demuestren el potencial de colonización endófito del hongo nematófago en otras especies de plantas de interés económico.

\section{Conclusiones}

Las cepas autóctonas cubanas IMI SD 187 (P. chlamydosporia var. catenula) y 193 (P. chlamydosporia var. chlamydosporia) colonizaron al interior de las raíces en especies de las familias Solanaceae y Cucurbitaceae, de importancia para cultivos protegidos de hortalizas.

El hongo nematófago bio-estimuló la longitud de la raíz en C. melo, así como, la cantidad de hojas y la masa fresca radical en $C$. апnиит en comparación con las plantas no inoculadas.

Además de utilizar al hongo nematófago en el manejo de nematodos fitoparásitos, se sugiere su evaluación en la bio-estimulación del crecimiento de otras especies de plantas de interés económico, lo que posibilitaría ampliar los usos agrícolas de la especie fúngica nematófaga.

\section{Referencias}

Arévalo, J., Hernández, M. Á., Lamz, A., Montes de Oca, N., \& Hidalgo-Díaz, L. (2019). Efecto de Pochonia chlamydosporia var. catenulata (Goddard) Zare y Gams como endófito facultativo en frijol (Phaseolus vulgaris L.). Revista Protección Vegetal, 34(2), 1-10.

Avelar, T. S., Vasconcelos, S., Ney, I., Coutinho, B., Megumi, M. C., de Araújo, J. V., \& de Freitas, L. (2018). Nematophagus fungi increasing phosphorus uptake and promoting plant growth. Biological Control, 123, 71-75. https://doi. org/10.1016/j.biocontrol.2018.05.003

Bordallo, J. J., López-Llorca, L. V., Jansson, H. B., Salinas, J., Persmark, L., \& Asensio, L. (2002). Colonization of plant roots by egg-parasitic and nematode-trapping fungi. New Phytologist, 154, 491-499.

Di-Rienzo, J. A., Casanoves, F., González, L., Tablada, M., \& Roblejo, C. W. (2008). InfoStat, versión 2008. Manual del Usuario. Grupo InfoStat, FCA, Universidad Nacional de Córdoba (2 ${ }^{\mathrm{da}}$ Ed.). Editorial Brujas Argentina.

Ghahremani, Z., Escudero, N., Saus, E., Gabaldón, T., \& Sorribas, F. J. (2019). Pochonia chlamydosporia induces plantdependent systemic resistance to Meloidogyne incognita. Frontiers in Plant Science, 10, Article 945. https://doi. org/10.3389/fpls.2019.00945

Gutiérrez, E., Rodríguez, J. L., \& Rivero, M. (2018). Flora del bosque pluvial montano y su relación con variables ambientales en el Parque Nacional Turquino de Cuba. Ciencia y Tecnología, 11(2), 57-67. https://doi.org/10.18779/cyt.v11i2.240

Hernández, M. A., Arévalo, J., Marrero, D., \& Hidalgo-Díaz, L. (2016). Efecto de KlamiC® en la estimulación del crecimiento de vitroplantas de plátanos y bananos. Cultivos Tropicales, 37(4), 168-172. https://doi.org/10.13140/ RG.2.2.25696.69120 
Hidalgo-Díaz, L., \& Ceiro, W. G. (2017). Interacción entre Pochonia chlamydosporia var. catenulata (Kamyschko ex Barron y Onions) Zare y Gams y Meloidogyne incognita (Kofoid y White) Chitwood en tomate en presencia de NaCl. Revista de Protección Vegetal, 32(1), 76-81.

Hidalgo-Díaz, L., Franco-Navarro, F., \& Freitas, L. G. (2017). Pochonia chlamydosporia microbial products to manage plantparasitic Nematodes: Case Studies from Cuba, Mexico \& Brazil. In R. H. Manzanilla-López, \& L. V. López-Llorca (Eds.), Perspectives in sustainable nematode management through Pochonia chlamydosporia Applications for root and rhizosphere health, sustainability in plant and crop protection ( ${ }^{\text {st }}$ Ed., 311-342), Springer, Chan. https://doi. org/10.1007/978-3-319-59224-4_15

Kerry, B. R., \& Bourne, J. M. (Eds.). (2002). A Manual for Research on Verticillium chlamydosporium, a Potential Biological Control Agent for Root-Knot Nematodes. University of Gent.

Kerry, B. R., \& Hirsch, P. R. (2011). Ecology of Pochonia chlamydosporia in the rhizosphere at the population, whole organism and molecular scales. In K. Davies, \& Y. Spiegel (Eds.). Biological Control of Plant-Parasitic Nematodes. Progress in Biological Control (pp. 171-182). Springer, Dordrecht. https://doi.org/10.1007/978-1-4020-9648-8_7

Latz, M. A. C., Jensen, B., Collinge, D. B., \& Jørgensen, H. J. L. (2018). Endophytic fungi as biocontrol agents: elucidating mechanisms in disease suppression. Plant Ecology and Diversity, 11(5-6), 555-567. https://doi.org/10.1080/175508 74.2018 .1534146

López-Llorca, L. V., Bordallo, J. J., Salinas, J., Monfort, E., \& Lopez-Serna, M. L. (2002). Use of light and scanning electron microscopy to examine colonisation of barley rhizosphere by the nematophagous fungus Verticillium chlamydosporium. Micron, 33(1), 61-67. https://doi.org/10.1016/S0968-4328(00)00070-6

López-Llorca, L. V., \& Maciá-Vicente, J. G. (2009). Plant symbioses with fungal endophytes: perspectives on conservation and sustainable exploitation of mediterranean ecosystems. Mediterranea: Serie de Estudios Biológicos, 2(20), 10-41.

Maciá-Vicente, J. G., Rosso, L. C., Ciancio, A., Jansson, H. B., \& López-Llorca, L. V. (2009). Colonisation of barley roots by endophytic Fusarium equiseti and Pochonia chlamydosporia: effects on plant growth and disease. Annals of Applied Biology, 155(3), 391-401. https://doi.org/10.1111/j.1744-7348.2009.00352.x

Montes de Oca, N., Arévalos, J., Nuñez, A., Riverón, Y., Villoch, A., \& Hidalgo-Díaz, L. (2009). KlamiC@: Experiencia técnicaproductiva. Revista de Protección Vegetal, 24(1), 62-65.

Nassimi,Z., \& Taheri, P. (2017). Endophytic fungus Piriformospora indica induced systemic resistance against rice sheath blight via affecting hydrogen peroxide and antioxidants. Biocontrol Science and Technology, 27(2), 252-267. https://doi.org /10.1080/09583157.2016.1277690

Rushda, S., \& Ambreen, A. (2014). Combined effect of biofertilizers and fertilizer in the management of Meloidogyne incognita and also on the growth of red kidney bean (Phaseolus vulgaris). International Journal of Plant Pathology, 5(1), 1-11. https://doi.org/10.3923/ijpp.2014.1.11

Shah, S., Shrestha, R., Maharjan, S., Selosse, M. A., \& Pant, B. (2019). Isolation and characterization of plant growth-promoting endophytic fungi from the roots of Dendrobium moniliforme. Plants, 8(5), 1-11. https://doi:10.3390/plants8010005

Siddiqui, Z. A., \& Akhtar, M. S. (2008). Synergistic effects of antagonistic fungi and a plant growth promoting rhizobacterium, an arbuscular mycorrhizal fungus, or composted cow manure on populations of Meloidogyne incognita and growth of tomato. Biocontrol Science and Technology, 18(3), 279-290. https://doi.org/10.1080/09583150801896043 
Topalovi'c, O., Hussain, M., \& Heuer, H. (2020). Plants and associated soil microbiota cooperatively suppress plant-parasitic nematodes. Frontiers of Microbiology, 11, Article 313. https://doi:10.3389/fmicb.2020.00313

Uddin, M., Saifullah, A. M., Khan, W., \& Khan, B. (2019). Evaluation of Pochonia chlamydosporia (Goddard) isolates for suppression of Meloidogyne incognita, root-knot nematode of tomato. Journal of Agricultural Science, 11(5), 70-81. https://doi.org/10.5539/jas.v11n5p70

Vieira Dos Santos, M. C., Curtis, R. H., \& Abrantes, I. (2014). The combined use of Pochonia chlamydosporia and plant defence activators-a potential sustainable control strategy for Meloidogyne chitwoodi. Phytopathologia Mediterranea, 53(1), $66-74$.

Vyas, S., Nagori, R., \& Purohit, S. D. (2008). Root colonization and growth enhancement of micropropagated Feronia limonia (L.) Swingle by Piriformospora indica-a cultivable root endophyte. International Journal of Plant Development of Biology, 2(2), 128-132. 